\title{
To review or not to review
}

\author{
How do editors at Nature Computational Science evaluate papers before deciding on whether to send them out to \\ peer review?
}

ven though Nature Computational
Science is still in its infancy, having
published four issues in total so far, the computational science community has been expressing substantial interest in our journal, which we really appreciate and are very excited about. As a result of this growing interest, we have received many questions from researchers regarding the scope of the journal and the types of papers in which we are interested. These are very natural questions, especially considering that our readership is exceptionally multidisciplinary: different domains might have different expectations when it comes to the structure and contents of a manuscript. Therefore, we would like to take a moment to provide some clarity on how we look at and assess the papers that our community submits to our journal.

First of all, it is worth highlighting that our editorial team consists of professional, full-time editors with diverse scientific backgrounds and $\mathrm{PhD}$ degrees. This means that we are entirely dedicated to the editorial process, and that we don't have competing or financial interests that could potentially bias our decisions. The chief editor assigns a primary editor for every paper - the assignment is based on which editor has the most relevant expertise - and the editor will read the paper and discuss it with at least one other editor at Nature Computational Science. And yes, we read your papers! A decision is then made on whether to send the paper out to review or to reject it without review.

But how is such a decision actually made? We are mostly interested in the development of new computational/ mathematical methods and software tools with the goal of addressing complex, relevant problems across a range of scientific disciplines. We also welcome applications of existing methods, but these applications should normally come with methodological development; for instance, straightforward usage of machine learning algorithms is usually outside of our scope. In terms of scientific disciplines, we have a list of our main topics of interests on the Aims \& Scope page, but this doesn't mean that we wouldn't consider other areas.

In addition to the main focus of a paper, we also assess its potential relevance to the domain of study. How important is the problem being addressed? What is the paper proposing (technically speaking, but also feature-wise) that is not already available in the literature? Does the approach have practical value? How does the approach compare with the state-of-the-art methods in the field? Does the paper provide a compelling experimental validation? The manuscript must have clear, sufficient information for the editors to properly evaluate these points. To guarantee immediate practical relevance, the authors must demonstrate the paper's potential impact by performing experiments with real data and by benchmarking against the most relevant related works, regardless of the main focus of the paper (method and/or software tool).

Finally, because our journal is multidisciplinary, we want our papers to reach a wide audience. What does this mean in practicality? First, we might give preference to papers that have potential impact across multiple disciplines. Second, we will try to diversify the topics we publish as much as we can to put forth our multidisciplinary nature. Last but not least, our papers must address the items mentioned in the previous paragraph, including having a clear motivation and experiments with real data, so that researchers from other fields can better understand the importance of the paper and the proposed methods, and potentially apply similar ideas to their own fields, thus creating this collaborative, diverse environment that we want to foster with our journal.

Everything from scope to potential relevance and practical value is then carefully taken into account by us when making an informed decision on a paper. This does not mean that we will only review a paper if it successfully addresses all of the aforementioned items: we might review a paper that looks very promising, even if it needs some work, or we might suggest some changes before we can send it out to review. If we reject a paper without review, it doesn't mean that it is a bad paper (trust us!): it might simply not reach our expected bar, or it might not be entirely appropriate to our audience, and, whenever possible, we will suggest other journals in the Nature Portfolio family that might be more appropriate.

We hope that this better clarifies what we look for in a manuscript before deciding on whether to review it or not. It is worth noting, however, that our editorial process does not stop there: if we select a paper for peer review, we will carefully oversee the entire review process, we will make a decision on the paper based on the submitted reviews, and if the paper is eventually accepted for publication, we will make sure that it follows our highest standards in reporting and reproducibility. One of our main goals at Nature Computational Science is to curate, disseminate, and improve the clarity and potential impact of high-quality work, and we will keep doing our best to provide an editorial service of quality to our community.

Published online: 26 April 2021 https://doi.org/10.1038/s43588-021-00068-1 